C.A. Díaz, F.A. Campos, J. Villar. Existence and uniqueness of conjectured supply function equilibria. International Journal of Electrical Power \& Energy Systems. vol. 58, pp. 266-273, June 2014. [Online: February 2014] JCR: 3.289 (2016)

\title{
Existence and Uniqueness of Conjectured Supply Function Equilibria
}

\author{
Cristian A. Díaz*, Fco. Alberto Campos, José Villar \\ Instituto de Investigación Tecnológica, Escuela Técnica Superior de Ingeniería, Universidad
} Pontificia Comillas, Madrid, Spain

*Alberto Aguilera 23, 28015 Madrid, Spain, cristian.diaz@iit.upcomillas

\begin{abstract}
Supply Function Equilibrium (SFE) and Conjectured Supply Function Equilibrium (CSFE) are some of the approaches most used to model electricity markets in the medium and long term.
\end{abstract}

SFE represents the generators' strategies with functions that link prices and quantities, but leads to systems of differential equations hard to solve, unless linearity is assumed (Linear Supply Function Equilibrium, LSFE). CSFE also assumes linearity of the supply functions but only around the equilibrium point, also avoiding the system of differential equations.

This paper analyzes the existence and uniqueness of G-CSFE (a CSFE previously proposed by the Authors) for both elastic and inelastic demands. In addition, it also proves that the iterative algorithm proposed to compute G-CSFE has a fixed point structure and is convergent, and that LSFE is a particular case of GCSFE when demand and marginal costs are linear. Selected examples show the performance of G-CSFE and how it can be applied to market power analysis with meaningful results.

Keywords: Conjectured supply function, supply function, inelastic demand, electricity market equilibrium, equilibrium existence and uniqueness.

\section{Introduction}

Several approaches have been proposed in the literature to compute and analyze the behavior of the generators in oligopolistic electricity markets (see [1] and [2]). Although most of these models have in common the simultaneous profit maximization of the generators (Nash equilibrium approach, see [3] and [4]), they differ in how the strategies of the generators are modeled. For example, strategies can be prices (Bertrand competition, see [5]), quantities (Cournot and Conjectural Variation approaches, see [6]-[9]), or supply functions, relating prices and quantities (Supply Function [10]-[12] and Conjectured Supply Function approaches [2], [13] and [14]). 
C.A. Díaz, F.A. Campos, J. Villar. Existence and uniqueness of conjectured supply function equilibria. International Journal of Electrical Power \& Energy Systems. vol. 58, pp. 266-273, June 2014. [Online: February 2014] JCR: 3.289 (2016)

Conjectured Supply Function Equilibrium (CSFE) is a combination of the Conjectural Variation and the Supply Function approaches, where the generators' strategies are represented by first order local approximations around the equilibrium point [2]. For each generator its conjecture is the slope of its residual demand curve (conjecture price-response, see [15] and $[16])^{1}$, which, at the equilibrium point, is calculated from the slopes of the first order approximations of its competitors bidding curves (see [2], [15] and [17]). Conjectures internalize most of the strategic behavior of the generators by representing their influence on the market price [16], but their computation depends on the CSFE approach selected, since the parameters of the linear approximations are sometimes supposed partially known (slope known and intercept unknown or vice versa), or totally unknown [2].

Knowing the slopes is equivalent to knowing the conjectures, since the latter can be directly computed from the former (see [14] and [15]). Very often these conjectures are estimated from historic generator bidding curves (see [7], [15] and [18]) and kept constant for all the forecasting horizon. However its use for medium and long term in a dynamic environment is rather arguable since conjectures should vary to reflect how the generators' strategies adapt to the evolving market structure and regulation (see [16] and [19]).

When the intercepts are given and the slopes are variables, the conjectures result from the equilibrium (see [2]) but are strongly conditioned by the given intercepts [16]. Therefore similar remarks as above about its applicability to medium and long term can be made.

When both intercepts and slopes are decision variables (G-CSFE), the supply functions of the generators are computed according to the market structure, and so are the conjectures. Thus, the generators strategies are dynamic and adapt to the market evolution, being more suited for medium and long term studies. However additional hypothesis must be considered to get a well-

1 Strictly talking, conjectures represent any assumption about the behaviour of the competitors [16]. However, in this specific case we assume that the conjecture, for a generator, corresponds to the slope of its residual demand slope (conjecture price-response) which, at the equilibrium, is consistent with the slopes of the supply function of its competitors [15]. 
C.A. Díaz, F.A. Campos, J. Villar. Existence and uniqueness of conjectured supply function equilibria. International Journal of Electrical Power \& Energy Systems. vol. 58, pp. 266-273, June 2014. [Online: February 2014] JCR: 3.289 (2016)

defined problem [16]. Reference [13] presents an efficient formulation for the G-CSFE and proposes an iterative algorithm to solve it. In addition, unlike the Linear Supply Function Equilibrium (LSFE, see [11], [20] and [21]), if the G-CSFE is solved for different demand values, a complete supply function, not necessarily linear, can be obtained for each generator (see [2] and [9]). However existence and uniqueness of the G-CSFE has not yet been analyzed. While the elastic case, under the assumption that both the marginal costs and the system demand are linear functions, can be considered already studied in [22], this article proves that this result cannot be directly applied to the inelastic demand case. On the contrary, specific considerations must be made to guarantee the existence and uniqueness of the solution.

It is important to emphasize that perfectly inelastic demand assumption for medium and long term analysis is a very common and sensible approach for avoiding the estimation of the demand elasticity in scenarios, see [23], where the future demand does not change as much as the market price does (see [24] and [9]).

This article has three main objectives. It proves that the G-CSFE includes, as a particular case, the LSFE when both the marginal costs and the elastic system demand are linear functions. Unlike [25] where only the symmetric case was analyzed, it also determines the conditions for the existence and uniqueness of the G-CSFE for the asymmetric case with both elastic and inelastic demands, proving that the inelastic case is not a particular case of the elastic one. Finally it determines the mathematical conditions under which the iterative algorithm proposed in [13] to compute the G-CSFE converges to the Nash-equilibrium, and presents some illustrative examples that show the applicability of the proposed algorithm to the computation of the market power of the generators (see [26] for a review on market power indices).

The article is organized as follows. Section 2 presents the formulation of the LSFE approach outlined in [11]. Section 3 describes the G-CSFE proposed in [13] for both elastic and inelastic demand representation, and proves that, when the demand and the marginal costs are linear, the LSFE is a particular case of the G-CSFE. Section 4 proves the existence and uniqueness 
C.A. Díaz, F.A. Campos, J. Villar. Existence and uniqueness of conjectured supply function equilibria. International Journal of Electrical Power \& Energy Systems. vol. 58, pp. 266-273, June 2014. [Online: February 2014] JCR: 3.289 (2016)

of the G-CSFE when the marginal costs are supposed to be linear-increasing. Section 5 tries to generalize the above result by extending it to the case of nonlinear-increasing marginal costs showing that in this case is not possible to guarantee the existence and uniqueness in a general way. Section 6 presents the necessary conditions under which the iterative algorithm proposed in [13] to compute the G-CSFE reaches the Nash-equilibrium. Section 7 discusses some illustrative examples about the equilibrium's existence showing the strong relationship between the conjectures and the structure of the market. Finally, Section 8 presents the main conclusions.

\section{Linear supply function equilibrium}

In the LSFE the production of the $i$ th generator $P_{i}$ is linearly related with its offer price $\pi_{i}$ in the following way:

$$
P_{i}\left(\pi_{i}\right)=\alpha_{i} \cdot\left(\pi_{i}-\gamma_{i}\right) \quad \forall i \in E
$$

$E$ being the set of generators, $\alpha_{i} \geq 0$ the slope of the function $\left(\alpha_{i}=\partial P_{i} / \partial \pi_{i}\right)$ and $\gamma_{i}$ the minimum offer price $\left(\pi_{i} \geq \gamma_{i}\right)$, both being decision variables.

The generation's costs are supposed to be quadratic, that is:

$$
C_{i}\left(P_{i}\left(\pi_{i}\right)\right)=\frac{1}{2} \cdot c_{i} \cdot\left(P_{i}\left(\pi_{i}\right)\right)^{2}+b_{i} \cdot P_{i}\left(\pi_{i}\right)+a_{i} \quad \forall i \in E
$$

$c_{i}, b_{i}$ and $a_{i}$ being the fixed coefficients of the costs' functions, leading to linear marginal costs.

The balance between the total production and the elastic system demand $D(\pi)$ is given

by:

$$
D(\lambda)=\sum_{i \in E} P_{i}(\lambda)
$$


C.A. Díaz, F.A. Campos, J. Villar. Existence and uniqueness of conjectured supply function equilibria. International Journal of Electrical Power \& Energy Systems. vol. 58, pp. 266-273, June 2014. [Online: February 2014] JCR: 3.289 (2016)

where $\lambda$ is the equilibrium market price and $\partial D(\pi) / \partial \pi=-\alpha^{0}, \alpha^{0}$ being the demand's elasticity ${ }^{2}$ and $\pi$ the price that consumers are willing to pay for a certain amount $D$.

The profit $B_{i}(\lambda)$ for each generator $i$ is given by:

$$
B_{i}(\lambda)=\lambda \cdot P_{i}(\lambda)-C_{i}\left(P_{i}(\lambda)\right) \quad \forall i \in E
$$

where $\lambda \cdot P_{i}(\lambda)$ represents the incomes and $C_{i}\left(P_{i}(\lambda)\right)$ the costs.

First order equilibrium conditions are obtained by deriving (4) with respect to the price $\lambda$ (decision variable for the LSFE, see [11]) and setting equal to zero, that is:

$$
\begin{aligned}
& \frac{\partial B_{i}(\lambda)}{\partial \lambda}=P_{i}(\lambda)+\lambda \cdot \frac{\partial P_{i}(\lambda)}{\partial \lambda}-\frac{\partial C_{i}\left(P_{i}(\lambda)\right)}{\partial P_{i}} \cdot \frac{\partial P_{i}(\lambda)}{\partial \lambda}=0 \\
& \Rightarrow P_{i}(\lambda)+\frac{\partial P_{i}(\lambda)}{\partial \lambda} \cdot\left(\lambda-\frac{\partial C_{i}\left(P_{i}(\lambda)\right)}{\partial P_{i}}\right)=0 \quad \forall i \in E
\end{aligned}
$$

where the derivative of the generator production with respect to the market price is the slope of its supply function, that is:

$$
\begin{aligned}
& P_{i}(\pi)=D(\pi)-\sum_{j \neq i} P_{j}(\pi) \\
& \left.\Rightarrow \frac{\partial P_{i}(\pi)}{\partial \pi}\right|_{\pi=\lambda}=\frac{\partial P_{i}(\lambda)}{\partial \lambda}=-\alpha^{0}-\sum_{j \neq i} \alpha_{j}
\end{aligned}
$$

Substituting (1), (2) and (6), into (5) leads to:

$$
\alpha_{i} \cdot\left(\lambda-\gamma_{i}\right)=\left(\alpha^{0}+\sum_{j \neq i} \alpha_{j}\right) \cdot\left(\lambda-\left(c_{i} \cdot\left(\alpha_{i} \cdot\left(\lambda-\gamma_{i}\right)\right)+b_{i}\right)\right) \quad \forall i \in E
$$

In the LSFE the supply functions of the generators are assumed to be consistent across all times (see [20]), which implies that (7) must be valid for any realization of $\lambda$. Equating coefficients of $\lambda$ :

$$
\alpha_{i}=\left(\alpha^{0}+\sum_{j \neq i} \alpha_{j}\right) \cdot\left(1-c_{i} \cdot \alpha_{i}\right) \quad \forall i \in E
$$

\footnotetext{
${ }^{2}$ Strictly speaking the demand elasticity is $(\Delta D / D) /(\Delta \lambda / \lambda)=-(\lambda / D) \cdot \alpha^{0}$. However, here for simplicity the elasticity refers to the slope $\alpha^{0}$.
} 
C.A. Díaz, F.A. Campos, J. Villar. Existence and uniqueness of conjectured supply function equilibria. International Journal of Electrical Power \& Energy Systems. vol. 58, pp. 266-273, June 2014. [Online: February 2014] JCR: 3.289 (2016)

Equating coefficients of the constant term:

$$
-\alpha_{i} \cdot \gamma_{i}=\left(\alpha^{0}+\sum_{j \neq i} \alpha_{j}\right) \cdot\left(c_{i} \cdot \alpha_{i} \cdot \gamma_{i}-b_{i}\right) \quad \forall i \in E
$$

Substituting the right side of (8) only into the left side of (9) and simplifying yields (see [11] for more details):

$$
\gamma_{i}=b_{i} \quad \forall i \in E
$$

Equation (10) means that the minimum offer price of each generator is its minimum marginal costs. Dividing (9) by $-\gamma_{i}$ and clearing $\alpha_{i}$, with $b_{i}=\gamma_{i}$, leads to:

$$
\alpha_{i}=\frac{\alpha^{0}+\sum_{j \neq i} \alpha_{j}}{1+c_{i} \cdot\left(\alpha^{0}+\sum_{\substack{j \neq i \\ j \in E}} \alpha_{j}\right)} \forall i \in E
$$

Equation (11) is equivalent to solve (8) and (9), and provides, together with (10), the solution of the LSFE.

It is necessary to highlight that in (11) it is assumed that all the generators are participating at all the equilibrium points ${ }^{3}$ obtained varying $\lambda$, implying that their productions are non null, $P_{i}>0$, and therefore $\alpha_{i}>0$ [20]. Subsection 3.1 presents a generalization that allows that some generators be out of the market due to high costs.

\section{Description of the G-CSFE}

In this market formulation the generators' strategies are again assumed to be linear functions (see (1)), but, unlike the LSFE, they are only valid around the equilibrium point (first order Taylor approximations [2]).

\footnotetext{
${ }^{3}$ A generator participates at an equilibrium point when its production is not affected by active constraints or is not null because of high costs.
} 
C.A. Díaz, F.A. Campos, J. Villar. Existence and uniqueness of conjectured supply function equilibria. International Journal of Electrical Power \& Energy Systems. vol. 58, pp. 266-273, June 2014. [Online: February 2014] JCR: 3.289 (2016)

Since slopes $\alpha_{i}$ are not null at the equilibrium point $\left(\partial P_{i}\left(\pi_{i}\right) /\left.\partial \pi_{i}\right|_{\pi i=\lambda}=\alpha_{i}>0\right.$ for the generators that participate at the equilibrium, see previous section), then:

$$
\frac{\partial B_{i}(\lambda)}{\partial \lambda}=\frac{\partial B_{i}(\lambda)}{\partial P_{i}} \cdot \frac{\partial P_{i}(\lambda)}{\partial \lambda}=0 \Leftrightarrow \frac{\partial B_{i}(\lambda)}{\partial P_{i}}=0 \quad \forall i \in E
$$

Thus, from (4), the first order equilibrium conditions are (see [13], [16]):

$$
\frac{\partial B_{i}(\lambda)}{\partial P_{i}}=0 \Rightarrow \lambda=M C_{i}\left(P_{i}\right)+\theta_{i} \cdot P_{i} \quad \forall i \in E
$$

where $M C_{i}\left(P_{i}\right)=\partial C_{i}\left(P_{i}\right) / \partial P_{i}$ is the marginal cost function, $P_{i}=P_{i}(\lambda)$ is the supply function evaluated at the clearing price, and $\theta_{i}=-\partial \lambda \partial \partial P_{i}$ is the generators residual demand's slope at the equilibrium point, called conjecture price-response or conjecture, that can be calculated as follows (see [15]):

$$
\theta_{i}=-\frac{\partial \lambda}{\partial P_{i}(\lambda)}=-\frac{1}{\partial D(\lambda) / \partial \lambda-\sum_{j \neq i} \partial P_{j}(\lambda) / \partial \lambda} \quad \forall i \in E
$$

$\partial D(\lambda) / \partial \lambda$ being the slope of the system demand and $\partial P_{j}(\lambda) / \partial \lambda$ the slope of the supply function of the generator $j$, both evaluated at the equilibrium point.

\subsection{Elastic demand}

For elastic demand we have $\partial D(\pi) / \partial \pi=-\alpha^{0}$. Hence, the strategic behavior of the generators strongly depends on $\alpha^{0}$ [27]. Indeed, if $\alpha^{0}$ increases, equation (14) shows that all the conjectures decrease and thus the level of competition increases.

Replacing (14) with $\partial D(\lambda) / \partial \lambda=-\alpha^{0}$ into (13) and including the balance equation (3) with linear demand $D(\lambda)=D^{0}-\alpha^{0} \cdot \lambda, D^{0}$ being the intercept of this curve ${ }^{4}$, the equilibrium conditions become: 
C.A. Díaz, F.A. Campos, J. Villar. Existence and uniqueness of conjectured supply function equilibria. International Journal of Electrical Power \& Energy Systems. vol. 58, pp. 266-273, June 2014. [Online: February 2014] JCR: 3.289 (2016)

$$
\begin{aligned}
& \lambda=M C_{i}\left(P_{i}\right)+\frac{1}{\alpha^{0}+\sum_{j \neq i} \alpha_{j}} \cdot P_{i} \quad \forall i \in E \\
& D=D^{0}-\alpha^{0} \cdot \lambda \\
& D=\sum_{i} P_{i}
\end{aligned}
$$

Even if $D^{0}$ and $\alpha^{0}$ are known, (15) is undetermined with $2 \cdot|E|+2$ variables $\left(P_{i}, \alpha_{i}, \lambda\right.$ and $D$ ), and $|E|+2$ equations. To solve it, [13] proposes a robustness criterion consisting of local linear approximations of the supply functions valid for two close equilibria corresponding to two close enough demand scenarios (G-CSFE approach). These scenarios can be built varying $D^{0}$ such that $D(\pi, \varepsilon)=D^{0}(\varepsilon)-\alpha^{0} \cdot \pi$ with $\varepsilon \in \Lambda=\left\{\varepsilon_{1}, \varepsilon_{2}\right\}$ and $D^{0}\left(\varepsilon_{2}\right)>D^{0}\left(\varepsilon_{1}\right)$.

Considering this robustness criterion, $\alpha_{i}$ can be approximated as follows:

$$
\alpha_{i}=\frac{\partial P_{i}}{\partial \lambda} \approx \frac{\Delta P_{i} / \Delta D^{0}}{\Delta \lambda / \Delta D^{0}}=\frac{P_{i}\left(\varepsilon_{2}\right)-P_{i}\left(\varepsilon_{1}\right)}{\lambda\left(\varepsilon_{2}\right)-\lambda\left(\varepsilon_{1}\right)} \quad \forall i \in E^{*}
$$

$E^{*}$ being the subset of generators participating at the equilibrium point $\left(E^{*} \subseteq E\right)$.

From (16), if a generator is not producing due to high costs, then $P_{i}\left(\varepsilon_{2}\right)=P_{i}\left(\varepsilon_{1}\right)=0$ and the generator can be considered out of the market. Therefore,

$$
\alpha_{i}=0 \quad \forall i \notin E^{*}
$$

Extending (15) for the two demand scenarios and including (16) and (17), the final determined equilibrium equations are:

$$
\begin{aligned}
& \lambda(\varepsilon)=M C_{i}\left(P_{i}(\varepsilon)\right)+\frac{1}{\alpha^{0}+\sum_{\substack{j \neq i \\
j \in E^{*}}} \alpha_{j}} \cdot P_{i}(\varepsilon) \quad \forall i \in E, \forall \varepsilon \in \Lambda \\
& D(\varepsilon)=D^{0}(\varepsilon)-\alpha^{0} \cdot \lambda(\varepsilon) \quad \forall \varepsilon \in \Lambda \\
& D(\varepsilon)=\sum_{i} P_{i}(\varepsilon) \quad \forall \varepsilon \in \Lambda \\
& \alpha_{i}=\frac{P_{i}\left(\varepsilon_{2}\right)-P_{i}\left(\varepsilon_{1}\right)}{\lambda\left(\varepsilon_{2}\right)-\lambda\left(\varepsilon_{1}\right)} \quad \forall i \in E^{*} \\
& \alpha_{i}=0 \quad \forall i \notin E^{*}
\end{aligned}
$$


C.A. Díaz, F.A. Campos, J. Villar. Existence and uniqueness of conjectured supply function equilibria. International Journal of Electrical Power \& Energy Systems. vol. 58, pp. 266-273, June 2014. [Online: February 2014] JCR: 3.289 (2016)

Substituting $\lambda\left(\varepsilon_{1}\right)$ and $\lambda\left(\varepsilon_{2}\right)$ from the first equation of (18) into the two last ones, and assuming linear marginal $\operatorname{costs} M C_{i}\left(P_{i}\right)=c_{i} \cdot P_{i}+b_{i}$, it is possible to deduce that:

$$
\begin{aligned}
& \alpha_{i}=\frac{P_{i}\left(\varepsilon_{2}\right)-P_{i}\left(\varepsilon_{1}\right)}{\left(c_{i} \cdot P_{i}\left(\varepsilon_{2}\right)+b_{i}+\frac{1}{\alpha^{0}+\sum_{\substack{j \neq i \\
j \in E^{*}}} \alpha_{j}} \cdot P_{i}\left(\varepsilon_{2}\right)\right)-\left(c_{i} \cdot P_{i}\left(\varepsilon_{1}\right)+b_{i}+\frac{1}{\alpha^{0}+\sum_{\substack{j \neq i \\
j \in E^{*}}} \alpha_{j}} \cdot P_{i}\left(\varepsilon_{1}\right)\right)} \\
& \Rightarrow \alpha_{i}=\frac{P_{i}\left(\varepsilon_{2}\right)-P_{i}\left(\varepsilon_{1}\right)}{\left(P_{i}\left(\varepsilon_{2}\right)-P_{i}\left(\varepsilon_{1}\right)\right) \cdot\left(c_{i}+\frac{1}{\alpha^{0}+\sum_{\substack{j \neq i \\
j \in E^{*}}} \alpha_{j}}\right)} \forall i \in E^{*}
\end{aligned}
$$

And therefore:

$$
\begin{aligned}
& \alpha_{i}=\frac{\alpha^{0}+\sum_{\substack{j \neq i \\
j \in E^{*}}} \alpha_{j}}{1+c_{i} \cdot\left(\alpha^{0}+\sum_{\substack{j \neq i \\
j \in E^{*}}} \alpha_{j}\right)} \forall i \in E^{*} \\
& \alpha_{i}=0 \quad \forall i \notin E^{*}
\end{aligned}
$$

Note that (20) is a more general formulation than (11) since (20) matches (11) when $E^{*}=E$. Note also that the strategy of a generator only takes into account the competitors that are not out of the market (set $\left.E^{*}\right), E^{*}$ being an equilibrium result.

Equation (20) also shows that with linear marginal costs the final slopes $\alpha_{i}$ (and therefore the conjectures, see (14)) do not depend on the productions $P_{i}(\varepsilon)$ and prices $\lambda(\varepsilon)$. Therefore $\alpha_{i}=\alpha_{i}(\varepsilon)$ is also independent of the demand scenarios $\varepsilon$. In addition, clearing $\gamma_{i}$ from (1) and evaluating it at $\lambda(\varepsilon)$ :

$$
\gamma_{i}(\varepsilon)=\lambda(\varepsilon)-P_{i}(\varepsilon) \cdot \frac{1}{\alpha_{i}} \quad \forall i \in E^{*}, \varepsilon \in \Lambda
$$

Applying the first equation of (18) to evaluate $\lambda(\varepsilon)$ also with linear marginal costs leads to: 
C.A. Díaz, F.A. Campos, J. Villar. Existence and uniqueness of conjectured supply function equilibria. International Journal of Electrical Power \& Energy Systems. vol. 58, pp. 266-273, June 2014. [Online: February 2014] JCR: 3.289 (2016)

$$
\gamma_{i}(\varepsilon)=P_{i}(\varepsilon) \cdot\left[c_{i}+\frac{1}{\alpha^{0}+\sum_{\substack{j \neq i \\ j \in E^{*}}} \alpha_{j}}-\frac{1}{\alpha_{i}}\right]+b_{i} \quad \forall i \in E^{*}
$$

Clearing $1 / \alpha_{i}$ from (20) and substituting it in (22), the bracketed term of (22) is null. Thus $\gamma_{i}(\varepsilon)=\gamma_{i}=b_{i}$ is also independent of $\varepsilon$ and corresponds to the solution of (10). Therefore, it is possible to state that the G-CSFE with linear system demand and linear marginal costs matches with the LSFE. Indeed, the methodology proposed in [13] is more general since it does not require linear marginal costs.

Note that prices $\lambda$ and productions $P_{i}$ for a certain demand curve $D(\pi)$ can be computed ${ }^{5}$ from (15) using $\alpha_{i}$ from (20).

\subsection{Inelastic/Invariant demand}

When the demand is invariant against changes in the market price, $\partial D(\pi) / \partial \pi=0 \Leftrightarrow D(\pi)=D^{0}$, the equilibrium conditions of the G-CSFE can be deduced from (18) with $\alpha^{0}=0$. If linear marginal costs are again considered, slopes $\alpha_{i}$ can be directly obtained by solving (20) with $\alpha^{0}=0$ (see the appendix of [13]), which substituted into (15) (also with $\alpha^{0}=0$ ) allows to compute the price and productions for a demand scenario. Note that inelastic demand does not necessarily mean inelastic residual demand.

\footnotetext{
${ }^{5}$ Reference [15] proves that with $\alpha_{i}$ fixed and convex cost functions, (15) can be computed using an equivalent quadratic optimization problem.
} 
C.A. Díaz, F.A. Campos, J. Villar. Existence and uniqueness of conjectured supply function equilibria. International Journal of Electrical Power \& Energy Systems. vol. 58, pp. 266-273, June 2014. [Online: February 2014] JCR: 3.289 (2016)

\section{Existence and uniqueness of the G-CSFE with linear increasing marginal cost functions}

\subsection{Elastic demand}

Section 3.1 showed that, when both the marginal costs and the demand are linear, the G-CSFE reduces to the LSFE and also meets the consistent conjectural equilibrium presented in [22]. Therefore, the existence and uniqueness of the G-CSFE for this particular case can be considered already proved in [22] and [28] (with $\alpha_{i}=1 / x_{i}$ and $\alpha^{0}=1 / f$ ).

\subsection{Inelastic/Invariant demand}

Section 3.2 mentioned that, with linear marginal costs, the slopes $\alpha_{i}$ for the inelastic case can be directly obtained by solving (20) with $\alpha^{0}=0$, which becomes (the slopes of generators out of $E^{*}$ are neglected since they are always null):

$$
\alpha_{i}=\frac{\sum_{\substack{j \neq i \\ j \in E^{*}}} \alpha_{j}}{1+c_{i} \cdot \sum_{\substack{j \neq i \\ j \in E^{*}}} \alpha_{j}} \quad \forall i \in E^{*}
$$

However, (23) is not a particular case of the elastic situation, and next sub-sections show that results of [22] and [28] for the elastic case cannot be used with $\alpha^{0}=0$, and specific considerations are needed to ensure existence and uniqueness of $\alpha_{i}$ and therefore of the equilibrium.

\subsubsection{Determination of $\alpha_{i}$}

If $y=\sum_{i \in E}^{*} \alpha_{i}$ is defined, solving (23) is equivalent to solving:

$$
\begin{aligned}
\alpha_{i} & =\frac{y-\alpha_{i}}{1+c_{i} \cdot\left(y-\alpha_{i}\right)} \quad \forall i \in E^{*} \\
y & =\sum_{i \in E^{*}} \alpha_{i}
\end{aligned}
$$


C.A. Díaz, F.A. Campos, J. Villar. Existence and uniqueness of conjectured supply function equilibria. International Journal of Electrical Power \& Energy Systems. vol. 58, pp. 266-273, June 2014. [Online: February 2014] JCR: 3.289 (2016)

Transforming $\alpha_{i}$ of (24) into a quadratic form:

$$
c_{i} \cdot\left(\alpha_{i}\right)^{2}-\left(c_{i} \cdot y+2\right) \cdot \alpha_{i}+y=0 \quad \forall i \in E^{*}
$$

the solutions of $\alpha_{i}$ are given by:

$$
\alpha_{i}=\frac{\left(c_{i} \cdot y+2\right) \pm \sqrt{\left(c_{i} \cdot y+2\right)^{2}-4 \cdot c_{i} \cdot y}}{2 \cdot c_{i}} \quad \forall i \in E^{*}
$$

Since $\alpha_{i} \geq 0$, assuming that the offer price of the generators must be greater or equal than their marginal costs for any production $P_{i}\left(\pi_{i} \geq M C_{i}\left(P_{i}\right)\right)$, then $\alpha_{i}$ must be located in the feasible region given by:

$$
0 \leq \alpha_{i} \leq \frac{1}{c_{i}} \quad \forall i \in E^{*}
$$

It is easy to prove that the only solution of (26) that satisfies (27) is ${ }^{6}$ :

$$
\alpha_{i}(y)=\frac{\left(c_{i} \cdot y+2\right)-\sqrt{\left(c_{i} \cdot y\right)^{2}+4}}{2 \cdot c_{i}} \quad \forall i \in E^{*}
$$

\subsubsection{Existence of at least one G-CSFE}

From (24) and (28), finding a solution for (23) is equivalent to solve the following system:

$$
\begin{aligned}
& \alpha_{i}=\alpha_{i}(y) \quad \forall i \in E^{*} \\
& y=\sum_{i \in E^{*}} \alpha_{i}
\end{aligned}
$$

where $\alpha_{i}(y)$ is the function defined in (28). In addition, from (24) and (27) it is possible to see that $y$ is bounded by:

$$
0 \leq y \leq \sum_{i \in E^{*}} \frac{1}{c_{i}}
$$

\footnotetext{
${ }^{6}$ The other solution has been discarded since the offer price could become less than the marginal cost.
} 
C.A. Díaz, F.A. Campos, J. Villar. Existence and uniqueness of conjectured supply function equilibria. International Journal of Electrical Power \& Energy Systems. vol. 58, pp. 266-273, June 2014. [Online: February 2014] JCR: 3.289 (2016)

Note that (29) has implicitly the structure of a fixed point problem $y=G(y)$, where from (28) $G(y)$ is:

$$
G(y)=\sum_{i \in E^{*}} \alpha_{i}(y)=\sum_{i \in E^{*}} \frac{\left(c_{i} \cdot y+2\right)-\sqrt{\left(c_{i} \cdot y\right)^{2}+4}}{2 \cdot c_{i}}
$$

According to Brouwer's fixed-point theorem (see [29]), if $G$ is a differentiable mapping defined in $I$ into itself, $G: I \rightarrow I$, with $I$ being a nonempty, compact and convex set, it is possible to state that there exists at least one fixed point $y^{*} \in I$ such that $G\left(y^{*}\right)=y^{*}$.

Note that from (31) the function $G$ is differentiable and from (27) it is defined in the next nonempty, compact and convex interval $I$ :

$$
I=\left[0, \sum_{i \in E^{*}} \frac{1}{c_{i}}\right]
$$

therefore $G(y)$ has at least a point $y^{*} \in I$ such that $G\left(y^{*}\right)=y^{*}$. Then, the equilibrium is obtained setting $\alpha_{i}=\alpha_{i}\left(y^{*}\right) \forall i \in E^{*}$.

\subsubsection{Uniqueness of the G-CSFE}

From (31) it can be seen that the fixed point $G(0)=0$ is a solution of (23) (see blue line in Figure 1). However, $\alpha_{i}=0 \forall i$ implies $P_{i}=0 \forall i$ (from (1)) and cannot be a solution for a non null demand $D^{0}>0$. Hence, it is necessary to guarantee that (31) has an additional single fixed point $y^{*}>0$. To do so the following conditions can be checked:

$$
\begin{gathered}
\frac{\partial G(y)}{\partial y}>0 \\
\frac{\partial^{2} G(y)}{\partial y^{2}}<0 \\
\left.\frac{\partial G(y)}{\partial y}\right|_{y=0}>1
\end{gathered}
$$

Indeed equations (33) and (34) imply that $G(y)$ is an increasing and concave function and equation (35) means that just after the fixed point $G(0)=0$ the function $G(y)$ (blue line in Figure 
C.A. Díaz, F.A. Campos, J. Villar. Existence and uniqueness of conjectured supply function equilibria. International Journal of Electrical Power \& Energy Systems. vol. 58, pp. 266-273, June 2014. [Online: February 2014] JCR: 3.289 (2016)

1) is larger than the line of fixed points $G(y)=y$ (red line in Figure 1). If all the above conditions hold simultaneously, then there exists only one fixed point $G\left(y^{*}\right)=y^{*}$ with $y^{*}>0$.

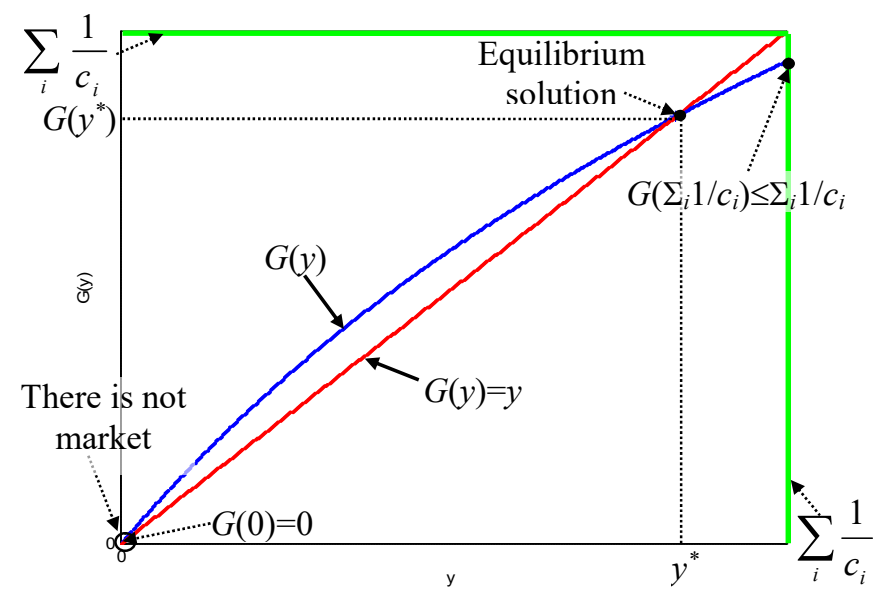

Figure 1. Equilibrium's solution for linear marginal costs and inelastic demand.

\section{Testing condition (33):}

Deriving $G(y)$ with respect to $y$ leads to:

$$
\frac{\partial G(y)}{\partial y}=\frac{1}{2} \cdot\left(\left|E^{*}\right|-\sum_{i \in E^{*}} \frac{c_{i} \cdot y}{\sqrt{\left(c_{i} \cdot y\right)^{2}+4}}\right)
$$

Since each term included into the summatory of (36) is less than one, condition (33) is satisfied.

Testing condition (34):

Using (36), $\partial^{2} G(y) / \partial y^{2}$ is given by:

$$
\frac{\partial^{2} G(y)}{\partial y^{2}}=-\frac{1}{2} \cdot \sum_{i \in E^{*}}\left(\frac{c_{i}}{\sqrt{\left(c_{i} \cdot y\right)^{2}+4}} \cdot\left(1-\frac{\left(c_{i} \cdot y\right)^{2}}{\left(c_{i} \cdot y\right)^{2}+4}\right)\right)
$$

Since $1>\left(c_{i} \cdot y\right)^{2} /\left(\left(c_{i} \cdot y\right)^{2}+4\right)$ in (37), then $G(y)$ is concave. 
C.A. Díaz, F.A. Campos, J. Villar. Existence and uniqueness of conjectured supply function equilibria. International Journal of Electrical Power \& Energy Systems. vol. 58, pp. 266-273, June 2014. [Online: February 2014] JCR: 3.289 (2016)

Evaluating (36) in $y=0$ leads to:

$$
\left.\frac{\partial G(y)}{\partial y}\right|_{y=0}=\left|E^{*}\right| \cdot \frac{1}{2}
$$

Thus condition (35) is only satisfied when $\left|E^{*}\right|>2$.

Therefore, for inelastic demand and linear marginal costs, if $\left|E^{*}\right|>2$, conditions (33)-(35) are satisfied simultaneously and the equilibrium exists and is unique. It is important to highlight that for elastic demand there is no minimum number of generators for the equilibrium existence.

When $\left|E^{*}\right|=2$, two alternatives exist. If $c_{1}=c_{2}$ (symmetric duopoly), there are infinite solutions for (23) whenever $\alpha_{1}=\alpha_{2}$. On the contrary, if $c_{1} \neq c_{2}$ (asymmetric duopoly), the only solution for (23) is $\alpha_{1}=\alpha_{2}=0$, leading to $P_{1}=P_{2}=0$. Note that this is the mathematical solution for (23) but it does not solve (15) for $D^{0}>0$.

If $\left|E^{*}\right|=1$ then the solution is a monopolistic situation where $\theta_{1} \rightarrow \infty$ and thus $\lambda \rightarrow \infty$. Again, although the above solution corresponds to the mathematical solution for (23), it does not correspond to an equilibrium problem since there is only one participant.

\section{Existence and uniqueness of the G-CSFE with nonlinear increasing marginal cost functions}

With linear marginal costs and the same minimum marginal costs for all the generators $\left(b_{i}=b_{j}\right.$ $\forall i \neq j)$, the aggregated marginal costs curve is also linear. This implies that, for any demand value $D, E^{*}=E$ and, from (11) and (23), the supply function slope $\alpha_{i}$ (and therefore $\theta_{i}$ ) is constant (corresponding to the LSFE). However, if nonlinear marginal costs, or different minimum marginal costs $\left(b_{i} \neq b_{j} \forall i \neq j\right)$ are considered (leading to a continuous linear piecewise aggregated marginal costs function), equation (20) can be rewritten as: 
C.A. Díaz, F.A. Campos, J. Villar. Existence and uniqueness of conjectured supply function equilibria. International Journal of Electrical Power \& Energy Systems. vol. 58, pp. 266-273, June 2014. [Online: February 2014] JCR: 3.289 (2016)

$$
\alpha_{i}=\frac{\alpha^{0}+\sum_{\substack{j \neq i \\ j \in E^{*}}} \alpha_{j}}{1+\frac{\partial M C_{i}\left(P_{i}\right)}{\partial P_{i}} \cdot\left(\alpha^{0}+\sum_{\substack{j \neq i \\ j \in E^{*}}} \alpha_{j}\right)} \quad \forall i \in E^{*}
$$

Hence, $\alpha_{i}$ cannot be considered invariant with the demand $D$. On the contrary, $\alpha_{i}$ depends on $\partial M C_{i}\left(P_{i}\right) / \partial P_{i}$ at the production $P_{i}$ and also on $E^{*}$, as shown in [13]. Indeed, system (39) is not well-defined since $\partial M C_{i}\left(P_{i}\right) / \partial P_{i} \forall i$ are also variables $(2 \cdot|E|$ variables with $|E|$ equations). Hence, one alternative to solve this problem is to join (15) and (39), resulting in a new nonlinear system for which we have not analyzed the existence and uniqueness of the solution yet. However, the iterative algorithm described in the next Section could still be used for the equilibrium computation, although it is not possible to guarantee the existence and uniqueness of the solution.

\section{Convergence of the algorithm for the G-CSFE}

From the previous two sections, if the marginal cost function of every generator is differentiable at the equilibrium point, then an equilibrium exists and is unique (with $\left|E^{*}\right|>2$ for inelastic demand, see section 4.2). If the marginal costs are linear, the equilibrium can directly be computed by solving (20) or (23) depending on the demand elasticity. However, for nonlinear increasing marginal costs, $\partial M C_{i}\left(P_{i}\right) / \partial P_{i}$ and $E^{*}$ are a priori unknown. In this case the iterative algorithm proposed in [13] and [30] can be applied for the equilibrium computation. This algorithm consists on a two-step iterative approach where, at each iteration $k$ : 
C.A. Díaz, F.A. Campos, J. Villar. Existence and uniqueness of conjectured supply function equilibria. International Journal of Electrical Power \& Energy Systems. vol. 58, pp. 266-273, June 2014. [Online: February 2014] JCR: 3.289 (2016)

- First step: solves two equilibria for two demand scenarios close enough (see (18)), with $\alpha_{i}$ and $E^{*}$ fixed $^{7}\left(\alpha_{i, k}\right.$ and $\left.E_{k}{ }^{*}\right)$, using the equivalent quadratic optimization problem of [15]. For elastic demand the objective function includes its utility [15]. This step provides the equilibrium productions $P_{i, k}(\varepsilon)$ and the marginal prices $\lambda_{k}(\varepsilon)$.

- Second step: computes $\alpha_{i, k+1}$ and determines $E_{k+1}{ }^{*}$ using $P_{i, k}(\varepsilon)$ and $\lambda_{k}(\varepsilon)$ from the first step as follows:

$$
\begin{aligned}
& \alpha_{i, k+1}=\frac{P_{i, k}\left(\varepsilon_{2}\right)-P_{i, k}\left(\varepsilon_{1}\right)}{\lambda_{k}\left(\varepsilon_{2}\right)-\lambda_{k}\left(\varepsilon_{1}\right)} \quad \forall i \in E \\
& E_{k+1}^{*}=\left\{i \in E: \alpha_{i, k+1}>0\right\}
\end{aligned}
$$

The algorithm converges when in two consecutive iterations the value of $\alpha_{i}$ does not change $\forall i$. Otherwise, the first step is executed again with the new values of $\alpha_{i}$.

Since the proposed algorithm has a structure of a fixed point algorithm (see Appendix) and there is at least a solution for (18), its convergence is guaranteed. However, convergence does not hold for inelastic demand with $\left|E^{*}\right| \leq 2$, or when $\partial M C_{i}\left(P_{i}\right) / \partial P_{i}$ does not exist.

\section{Numerical examples}

This section uses four examples to discuss the goodness of the iterative algorithm proposed in [13] and [30] to compute the G-CSFE with general marginal costs functions and inelastic demand $\left(D(\pi)=D^{0}\right)$. These examples consider both linear and nonlinear increasing marginal costs functions (cases A and B respectively).

\subsection{Linear marginal costs}

The following cases have been analyzed:

\footnotetext{
${ }^{7}$ If the solution of the problem to be solved exists and is unique, since the iterative algorithm used to solve it has a fixed point structure, the initial $\alpha_{i}$ and $E^{*}$ do not affect the numerical solution. The initial scenario may affect the number of iterations that the algorithm needs to find the equilibrium.
} 
C.A. Díaz, F.A. Campos, J. Villar. Existence and uniqueness of conjectured supply function equilibria. International Journal of Electrical Power \& Energy Systems. vol. 58, pp. 266-273, June 2014. [Online: February 2014] JCR: 3.289 (2016)

- $\quad$ Three generators with $b_{i}=0 \forall i$.

- $\quad$ Three generators with $b_{i}>0 \forall i$ and $b_{1} \neq b_{2} \neq b_{3}$.

- $\quad$ Five generators with $b_{i}>0 \forall i$ and $b_{1} \neq b_{2} \neq b_{3}=b_{4}=b_{5}$.

In all cases the initial values of $\alpha_{i}$ have been set to the slopes ci of the marginal costs functions.

\subsubsection{Three generators with $b_{i}=0 \forall i$}

Figure 2 depicts the marginal costs $M C_{1}\left(P_{1}\right)=0.020 \cdot P_{1}, M C_{2}\left(P_{2}\right)=0.001 \cdot P_{2}$ and $M C_{3}\left(P_{3}\right)=0.005 \cdot P_{3}$, and the supply functions (SFi) for all the generators. Likewise, Figure 3 shows the production of each generator (Absi), and its power share (p.u.i). Since $b_{l}=0 \forall i$, the aggregated marginal costs (MCT) and supply function (MCA) are linear (see Figure 2). Hence, the three generators have non null productions for any demand value and $E^{*}=E$.

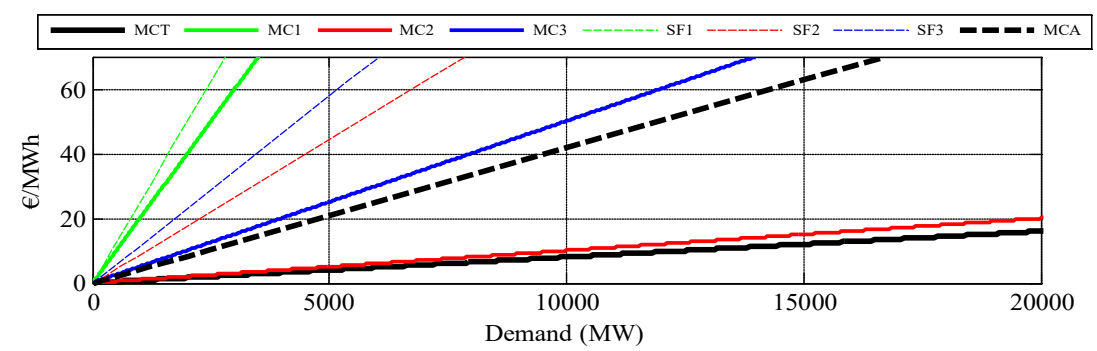

Figure 2. Marginal costs and supply functions for three generators and $b_{i}=0 \forall i$

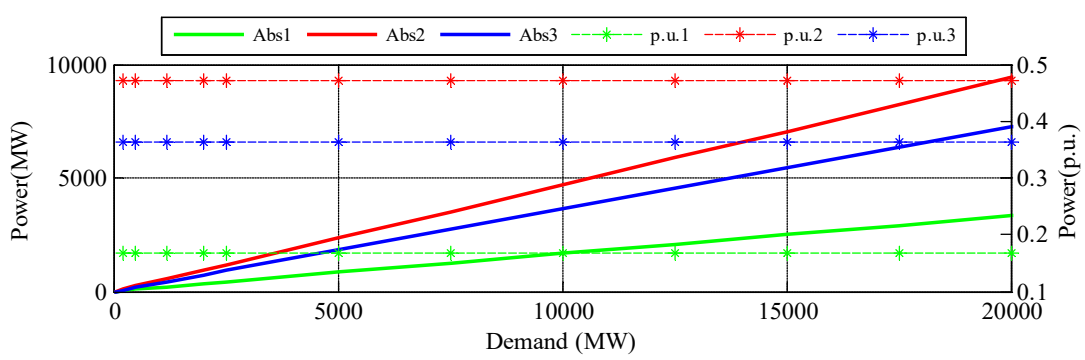

Figure 3. Distribution of the power for three generators and $b_{i}=0 \forall i$

The resulting power share (see p.u. $i$ in Figure 3) for each generator is inversely proportional to its marginal costs' slope (see $\mathrm{MCi}$ in Figure 2), and constant for any demand value. 
C.A. Díaz, F.A. Campos, J. Villar. Existence and uniqueness of conjectured supply function equilibria. International Journal of Electrical Power \& Energy Systems. vol. 58, pp. 266-273, June 2014. [Online: February 2014] JCR: 3.289 (2016)

Even if each solution can be computed directly by solving (23), this simple case study has been selected to show, in Figure 4, how the iterative algorithm of [13] evolves until convergence (after 15 iterations).

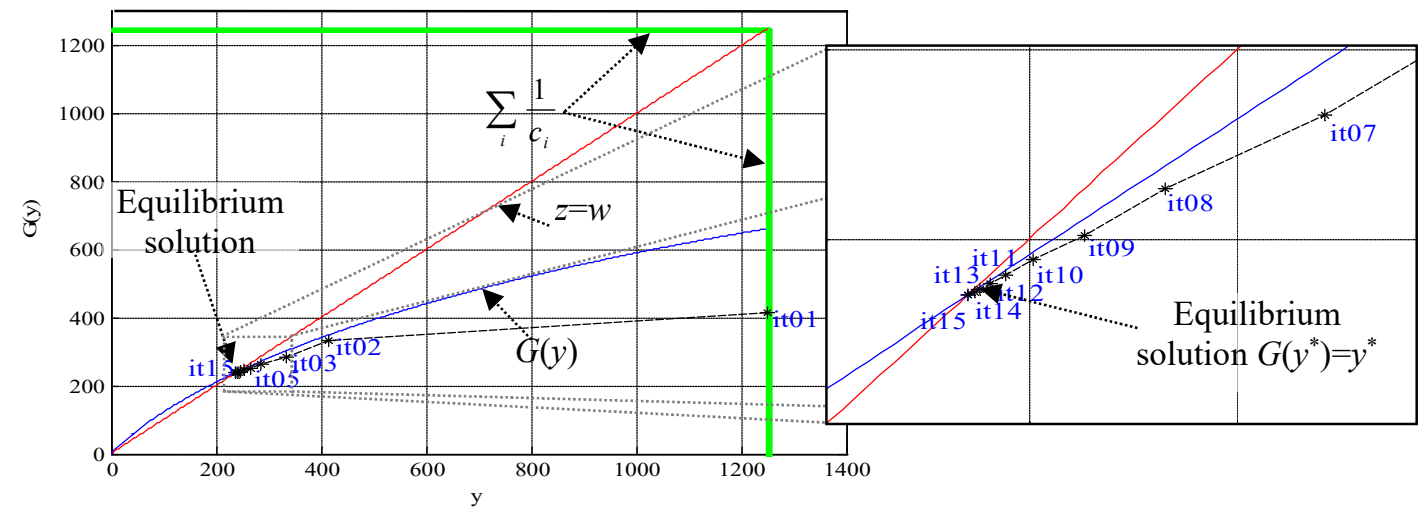

Figure 4. Equilibrium's solution for three generators with linear marginal costs and inelastic demand

\subsubsection{Three generators with $b_{i}>0 \forall i$ and $b_{1} \neq b_{2} \neq b_{3}$}

Figure 5 shows the marginal costs (MCi) and the supply functions ( $\mathrm{SF} i$ ) for all the generators of the case 7.1.1 when $b_{1}=30, b_{2}=40$ and $b_{3}=25$. Likewise, Figure 6 displays the cleared power (Abs $i$ ) and power share (p.u.i) for each one of them. As proved before, the equilibrium can only be computed when $\left|E^{*}\right|>2$.Therefore, since $b_{i} \neq b_{j} \forall i, j$, the generators' supply functions cannot be computed for all the demand range (see for example Figure 5 for prices lesser than $40 € / \mathrm{MWh}$ ).

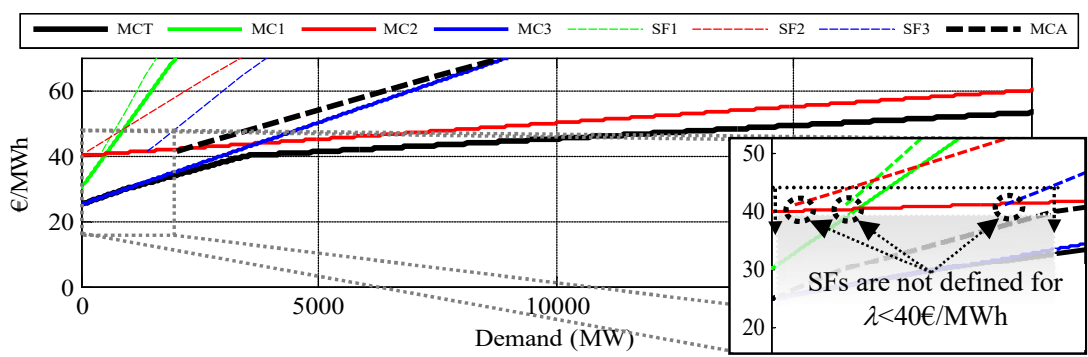

Figure 5. Marginal costs and supply functions for three generators and $b_{i} \neq b_{j} \forall i, j$ 
C.A. Díaz, F.A. Campos, J. Villar. Existence and uniqueness of conjectured supply function equilibria. International Journal of Electrical Power \& Energy Systems. vol. 58, pp. 266-273, June 2014. [Online: February 2014] JCR: 3.289 (2016)

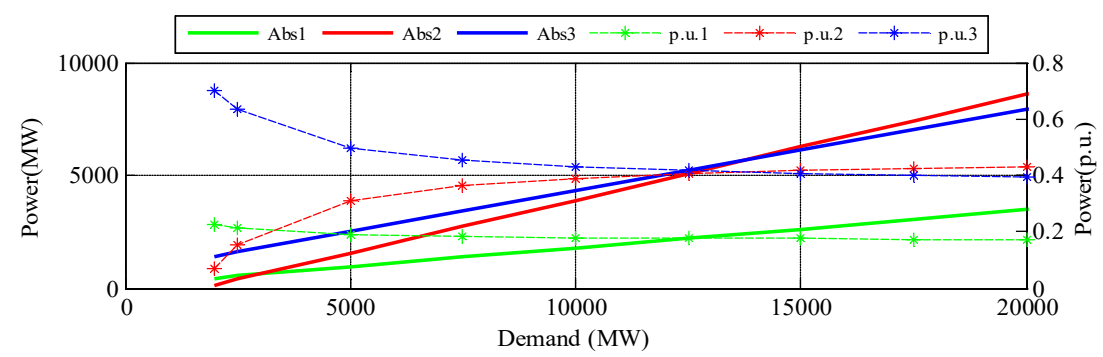

Figure 6. Distribution of the power for three generators and $b_{i} \neq b_{j} \forall i, j$

For prices larger than $40 € / \mathrm{MWh}$ the value of $\alpha_{i}$ remains constant since the marginal costs functions are linear and the number of generators into the market remains constant. However, this does not imply that the power share of the generators is constant too. Unlike the previous case where the power shares were only affected by $\alpha_{i}$, here they are also affected by the intercept of the marginal costs $b_{i}$ (for those demands where $\alpha_{i}$, the production of a generator can be computed as a function of the market price as $\left.P_{i}=\left(\lambda-b_{i}\right) / \alpha_{i}\right)$. For example, Figure 6 shows that for lower demands, generator 3 produces more than its competitors ( $b_{3}$ being the lowest). However, for higher demands, generator 2 has the largest power share and the system goes into a situation similar to case 7.1.1.

\subsubsection{Five generators with $b_{i}>0 \forall i$ and $b_{1} \neq b_{2} \neq b_{3}=b_{4}=b_{5}$}

The same three generators of case 7.1.2 have been used, and two additional generators with $M C_{4}\left(P_{4}\right)=0.010 \cdot P_{4}+25$ and $M C_{5}\left(P_{5}\right)=0.050 \cdot P_{5}+25$ have been included. Figure 7 shows the marginal costs $(\mathrm{MC} i)$ and the supply functions $(\mathrm{SF} i)$ for the 5 generators. Note that both new generators have the same minimum marginal costs as generator 3 (the lowest one). Therefore there are at least three generators producing for any demand level, and their supply functions are defined for every demand value. However, the number of generators into the market changes when the system demand increases. Therefore, supply functions' slopes are not constant. This reinforces the idea that supply functions should not be represented by a single linear function, but can be built from linear approximations for the full range of system demands (see [2], [9] and [13]). 
C.A. Díaz, F.A. Campos, J. Villar. Existence and uniqueness of conjectured supply function equilibria. International Journal of Electrical Power \& Energy Systems. vol. 58, pp. 266-273, June 2014. [Online: February 2014] JCR: 3.289 (2016)

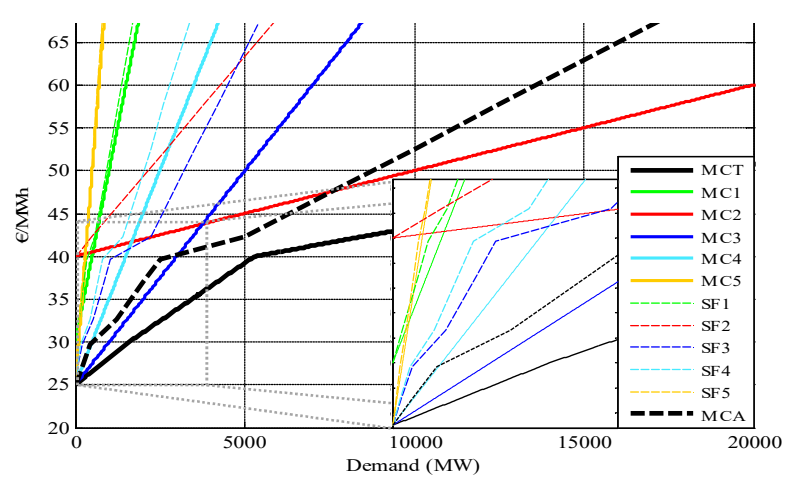

Figure 7. Marginal costs and supply functions with five generators

The effect of the number of the generators producing can also be seen in Figure 8 .

In this case the analysis reveals different price intervals. The first one corresponds to prices between 25 and $30 € / \mathrm{MWh}$, where only three generators (generators 3, 4 and 5) produce $\left(P_{i}>0\right)$ and their strategy remains constant. Then, for $40 € / \mathrm{MWh}>\lambda>30 € / \mathrm{MWh}$, generator 1 enters into the market and produces, modifying the behavior of the three initial generators, and leading to a more competitive situation. This fact can be seen in Figure 8, where the slope of the MCA decreases compared to the initial one (for $30 € / \mathrm{MWh}>\lambda>25 € / \mathrm{MWh}$ ). The final price interval is for $\lambda>40 € / \mathrm{MWh}$. From this point, all the generators are producing, implying new strategic changes and increasing competition.

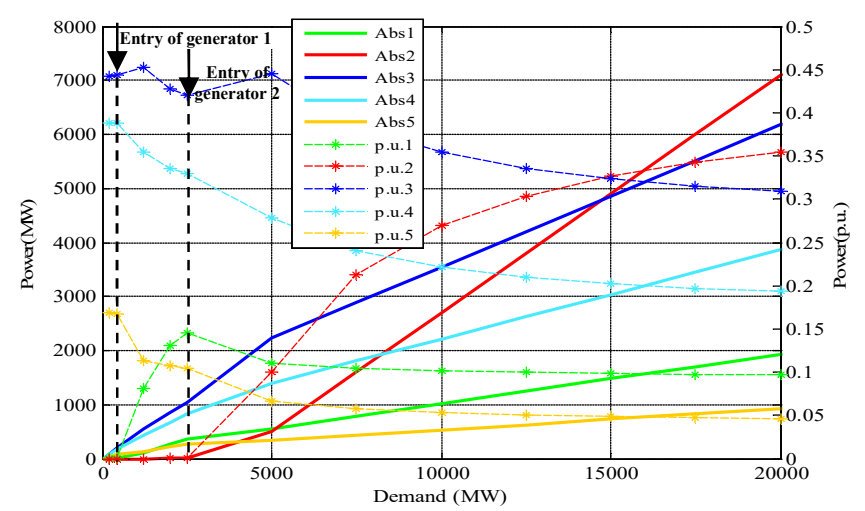

Figure 8. Distribution of the power with five generators

\subsection{Nonlinear marginal costs}

Five generators with the following marginal costs are considered: 
C.A. Díaz, F.A. Campos, J. Villar. Existence and uniqueness of conjectured supply function equilibria. International Journal of Electrical Power \& Energy Systems. vol. 58, pp. 266-273, June 2014. [Online: February 2014] JCR: 3.289 (2016)

- $M C_{1}\left(P_{1}\right)=1.98 \mathrm{e}-6 \cdot\left(P_{1}\right)^{2}+2.3 \mathrm{e}-3 \cdot P_{1}+30$

- $M C_{2}\left(P_{2}\right)=5.83 \mathrm{e}-7 \cdot\left(P_{2}\right)^{2}+4.237 \mathrm{e}-4 \cdot P_{3}+40$

- $M C_{3}\left(P_{3}\right)=1.98 \mathrm{e}-6 \cdot\left(P_{3}\right)^{2}+1.04 \mathrm{e}-3 \cdot P_{3}+25$

- $M C_{4}\left(P_{4}\right)=5.0 \mathrm{e}-6 \cdot\left(P_{4}\right)^{2}+2.36-3 \cdot P_{4}+25$

- $M C_{5}\left(P_{5}\right)=3.0 \mathrm{e}-7 \cdot\left(P_{5}\right)^{2}+5.0 \mathrm{e}-3 \cdot P_{5}+25$

The initial values of $\alpha_{i}$ have been set to the derivative of the marginal cost function evaluated at $P_{i}=0$, that is $\alpha_{i}=\partial M C_{i}\left(P_{i}\right) /\left.\partial P_{i}\right|_{P i=0 \text {. }}$

Figure 9 shows the marginal costs (MCi) and the supply functions (SFi) for the five generators. In this case the aggregated marginal cost function consists of three quadratic and continuous segments (MCT). The results show how the proposed iterative algorithm is able to compute the supply functions even when the marginal costs are not linear, the only requirement being that the equilibria occur at differentiable points of the aggregated marginal costs function (see [15]). Figure 9 also shows the extra cost (from the demand side) or extra offer prices (from the generators side) due to the conjectures mark-up, with respect to a pure marginal cost system.

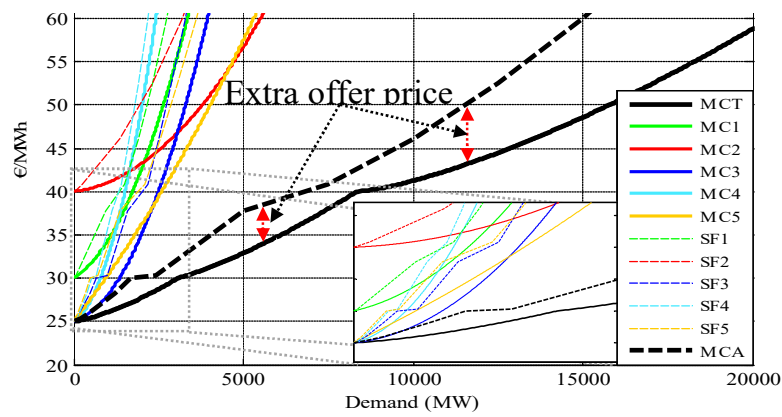

Figure 9. Nonlinear marginal costs and supply functions with five generators

\section{Conclusions}

This article presents the formulation of the Conjectured Supply Function Equilibrium proposed in [13] (denoted as G-CSFE) for elastic and inelastic system demand when general increasing (linear or nonlinear) marginal costs functions are considered. Likewise, if the system demand is elastic and the marginal costs are supposed to be linear, it shows that the well-known Linear 
C.A. Díaz, F.A. Campos, J. Villar. Existence and uniqueness of conjectured supply function equilibria. International Journal of Electrical Power \& Energy Systems. vol. 58, pp. 266-273, June 2014. [Online: February 2014] JCR: 3.289 (2016)

Supply Function Equilibrium (LSFE) and the G-CSFE are totally equivalent. Then, since for the G-CSFE the marginal costs functions are not necessarily linear, LSFE can be considered as a particular case.

Taking into account that in general the electricity demand elasticity is rather small and hard to estimate, the inelastic demand assumption is a very frequent approximation for medium and long term analysis. This article proves that G-CSFE exists and is unique for inelastic demand and linear marginal costs, when the number of generators producing is strictly greater than two, which significantly differs from the elastic case where the equilibrium exists for any number of generators. However, it is not possible a priori to know the equilibrium location, making the use of an iterative algorithm, such as the one proposed in [13] and [30], a very convenient approach. Finally this article also proves that the algorithm proposed in [13] and [30] has a fixed point structure and therefore is convergent.

Numerical examples show that, modeling the supply functions with linear functions strongly conditions the final solution, and seems only acceptable when all the generators have linear marginal costs functions with same minimum cost, but should be avoided for more realistic markets. Indeed, the shapes of the supply functions cannot be guessed a priori, but they can be built by means of linear approximations around the equilibrium point for all the possible equilibria obtained varying the demand, as it is proposed here and in [13] and [30].

\section{Appendix}

Let's prove that the algorithm of [13] is in fact a fixed point algorithm. On the one hand, the proposed algorithm computes the slopes $\alpha_{i, k+1}$ as follows:

$$
\begin{aligned}
& \alpha_{i, k+1}=\frac{P_{i, k}\left(\varepsilon_{2}\right)-P_{i, k}\left(\varepsilon_{1}\right)}{\lambda_{k}\left(\varepsilon_{2}\right)-\lambda_{k}\left(\varepsilon_{1}\right)} \quad \forall i \in E \\
& E_{k+1}^{*}=\left\{i \in E: \alpha_{i, k+1}>0\right\}
\end{aligned}
$$

where each $P_{i, k}$ is obtained solving (15) with $\alpha_{i}=\alpha_{i, k}$ and $E^{*}=E_{k}{ }^{*}$. 
C.A. Díaz, F.A. Campos, J. Villar. Existence and uniqueness of conjectured supply function equilibria. International Journal of Electrical Power \& Energy Systems. vol. 58, pp. 266-273, June 2014. [Online: February 2014] JCR: 3.289 (2016)

On the other hand, a standard fixed point algorithm computes $y^{k+1}=\sum_{i} \alpha_{i, k+1}^{\prime}$ such as

$y^{k+1}=G\left(y^{h}\right)$, being $G$ the mapping defined in (31) and $y^{k}=\sum_{i} \alpha_{i, k}$.

Note that $\alpha_{i, k+1}^{\prime}=\alpha_{i, k+1} \forall i$, since $\alpha_{i, k+1}^{\prime}$ satisfies (23) which has been deduced from the

last two equations of (18).

\section{References}

[1] M. Ventosa, A. Baillo, A. Ramos, and M. Rivier, “Electricity market modeling trends," Energy policy, vol. 33, no. 7, pp. 897-913, 2005.

[2] C. J. Day, B. F. Hobbs, and J. S. Pang, "Oligopolistic competition in power networks: a conjectured supply function approach,” IEEE Transactions on Power Systems, vol. 17, no. 3, pp. 597-607, 2002.

[3] J. F. Nash, "Equilibrium Points in N-Person Games," Proceedings of the National Academy of Sciences of the United States of America, vol. 36, no. 1, pp. 48 -49, 1950.

[4] D. Langary, N. Sadati, and A. M. Ranjbar, "Direct approach in computing robust Nash strategies for generating companies in electricity markets," International Journal of Electrical Power \& Energy Systems, vol. 54, pp. 442-453, Jan. 2014.

[5] K. G. Dastidar, "On the existence of pure strategy Bertrand equilibrium," Econ Theory, vol. 5, no. 1, pp. 19-32, Feb. 1995.

[6] C. Metzler, B. F. Hobbs, and J. S. Pang, "Nash-Cournot equilibria in power markets on a linearized DC network with arbitrage: Formulations and properties," Networks and Spatial Economics, vol. 3, no. 2, pp. 123-150, 2003.

[7] Y. Song, Z. Hou, F. Wen, Y. Ni, and F. F. Wu, “Conjectural variation based learning of generator's behavior in electricity market," in International Symposium on Circuits and Systems, 2003, vol. 3.

[8] M. K. Perry, "Oligopoly and consistent conjectural variations," The Bell Journal of Economics, vol. 13, no. 1, pp. 197-205, 1982.

[9] Y. Song, Y. Ni, F. Wen, and F. F. Wu, "Analysis of strategic interactions among generation companies using conjectured supply function equilibrium model," in IEEE Power Engineering Society General Meeting, 2003, vol. 2.

[10] S. Soleymani, "Bidding strategy of generation companies using PSO combined with SA method in the pay as bid markets," International Journal of Electrical Power \& Energy Systems, vol. 33, no. 7, pp. 1272-1278, Sep. 2011.

[11] R. Baldick, R. Grant, and E. Kahn, "Theory and application of linear supply function equilibrium in electricity markets," Journal of Regulatory Economics, vol. 25, no. 2, pp. 143-167, 2004.

[12] R. J. Green, "Increasing Competition in the British Electricity Spot Market," Journal of Industrial Economics, vol. 44, no. 2, pp. 205-16, 1996.

[13] C. A. Díaz, J. Villar, F. A. Campos, and M. A. Rodríguez, "A new algorithm to compute conjectured supply function equilibrium in electricity markets," Electric Power Systems Research, vol. 81, no. 2, pp. 384-392, 2011.

[14] J. Barquín, B. Vitoriano, E. Centeno, and F. Fernández-Menéndez, “An optimization-based conjectured supply function equilibrium model for network constrained electricity markets," Journal of the Operational Research Society, vol. 60, no. 12, pp. 1719-1729, 2008.

[15] J. Barquín, E. Centeno, and J. Reneses, "Medium-term generation programming in competitive environments: a new optimisation approach for market equilibrium computing," IEE Proceedings on Generation, Transmission and Distribution, vol. 151, no. 1, pp. 119-126, 2004.

[16] C. A. Díaz, J. Villar, F. A. Campos, and J. Reneses, "Electricity market equilibrium based on conjectural variations," Electric Power Systems Research, vol. 80, no. 12, pp. 1572-1579, 2010.

[17] J. D. Liu and T. T. Lie, "Empirical dynamic oligopoly behavior analysis in electricity markets," presented at the International Conference on Power System Technology, PowerCon., 2004, vol. 2, pp. 1768-1773.

[18] D. W. Bunn, Modelling Prices in Competitive Electricity Markets, 1st ed. Wiley, 2004. 
C.A. Díaz, F.A. Campos, J. Villar. Existence and uniqueness of conjectured supply function equilibria. International Journal of Electrical Power \& Energy Systems. vol. 58, pp. 266-273, June 2014. [Online: February 2014] JCR: 3.289 (2016)

[19] C. A. Díaz, F. A. Campos, and J. Villar, "Impact of the Electricity Contracts on the Generators' Strategic Behavior," in 9th International Conference on the European Energy Market, Florence, Italy, 2012.

[20] R. Baldick, R. Grant, and E. Kahn, "Linear Supply Function Equilibrium: Generalizations, Application, and Limitations," PWP-078, 2000.

[21] A. Rudkevich, "Supply function equilibrium: theory and applications," presented at the 36th International Conference on System Sciences, Hawaii, 2002.

[22] Y. Liu, Y. X. Ni, F. F. Wu, and B. Cai, "Existence and uniqueness of consistent conjectural variation equilibrium in electricity markets," International Journal of Electrical Power \& Energy Systems, vol. 29, no. 6, pp. 455-461, 2007.

[23] Z. Qiu, N. Gui, and G. Deconinck, "Analysis of equilibrium-oriented bidding strategies with inaccurate electricity market models," International Journal of Electrical Power \& Energy Systems, vol. 46, pp. 306-314, Mar. 2013.

[24] Y. Zhang, K. Tian, Y. Li, and M. Zeng, "Study on the Modeling of Power Market Based on Conjectured Supply Function Equilibrium," in 2008 International Conference on Risk Management \& Engineering Management, Beijing, China, 2008, pp. 328-333.

[25] P. Holmberg, "Unique supply function equilibrium with capacity constraints," Energy Economics, vol. 30 , no. 1 , pp. $148-172$, Jan. 2008.

[26] S. Prabhakar Karthikeyan, I. Jacob Raglend, and D. P. Kothari, “A review on market power in deregulated electricity market," International Journal of Electrical Power \& Energy Systems, vol. 48, pp. 139-147, Jun. 2013.

[27] E. Bompard, Y. Ma, R. Napoli, and G. Abrate, "The Demand Elasticity Impacts on the Strategic Bidding Behavior of the Electricity Producers," IEEE Transactions on Power Systems, vol. 22, no. 1, pp. 188-197, 2007.

[28] Y. Liu, Y. X. Ni, and F. F. Wu, "Existence, uniqueness, stability of linear supply function equilibrium in electricity markets," in IEEE Power Engineering Society General Meeting, 2004, vol. 1, pp. 249-254.

[29] Arizona University, "Fixed Point Theorems." [Online]. Available: http://www.u.arizona.edu/ mwalker/econ519/Econ519LectureNotes/FixedPointTheorems.pdf.

[30] C. A. Díaz, F. A. Campos, J. Villar, and M. A. Rodríguez, "Endogenous Computation of Conjectured Supply Functions with Network Constraints," Electric Power Systems Research, vol. 90, no. 0, pp. 117-125, 2012. 